\title{
ANÁLISE DO VOLUME DA CASCA EM PORCENTAGEM EM BRACATINGAIS DE REGENERAÇÃO NATURAL INDUZIDA DA REGIÃO METROPOLITANA DE CURITIBA, PR
}

\author{
Sebastião do Amaral Machado*, Luís César Rodrigues da Silva**, Edilson Urbano***, \\ Marco Aurélio Figura**, Saulo Jorge Téo**, Barbara Jarshel** \\ *Eng. Florestal, Dr., Depto. de Ciências Florestais, UFPR, Pesquisador do CNPq - sammac@floresta.ufpr.br \\ **Graduando em Eng. Florestal, UFPR - nabravo@floresta.ufpr.br; figura_floresta@hotmail.com; sauloteo@yahoo.com.br; \\ barbara floresta@yahoo.com.br \\ ***Eng. Florestal, Mestrando em Eng. Florestal, UFPR - edurbano2@hotmail.com
}

Recebido para publicação: 03/11/2005 - Aceito para publicação: 24/04/2006

\begin{abstract}
Resumo
O objetivo desta pesquisa foi testar a metodologia de Meyer, para calcular o volume da casca como uma porcentagem do volume total com casca e testar sua acuracidade, bem como verificar se o volume de casca em porcentagem é constante ao longo do fuste. Os dados provieram de 179 árvores de bracatinga (Mimosa scabrella Bentham) distribuídas em diferentes bracatingais de regeneração natural da região metropolitana de Curitiba, estado do Paraná. Todas as árvores foram derrubadas e cubadas segundo metodologia de Hohenadl. A cubagem forneceu o volume com casca e sem casca, podendo-se assim comparar os volumes da casca em porcentagem obtidos pela fórmula de Meyer com os valores reais obtidos pelo método tradicional da cubagem com e sem casca. Verificou-se que a fórmula introduzida por Meyer, usando medições de diâmetros apenas no DAP, subestimou o volume da casca em porcentagem para todas as classes de DAP e idades, e que a diferença foi significativa segundo as análises estatísticas realizadas. Verificou-se também que a relação de diâmetro sem casca sobre diâmetro com casca não permanece constante ao longo do fuste e conseqüentemente o volume da casca em porcentagem também não é constante.

Palavras-chave: Fórmula de Meyer; volume da casca; fator de casca; Mimosa scabrella.
\end{abstract}

\begin{abstract}
Bark volume in percent analysis in Mimosa scabrella from natural induced regeneration stands in Curitiba Metropolitan Region. The objective of this paper was to test Meyer's methodology to calculate bark volume as a percent of total outside bark volume and to see how acurate it is, as well as to test the hypothesis that the bark volume in percentage is constant along the stem. The data came from 179 Mimosa scabrella Bentham trees from spontaneous generation stands, located in the Curitiba metropolitan region, Paraná, Brazil. All trees were cut down and their diameters inside and outside bark were measured at different heights, according to Hohenadl's formula. The observed volume outside and inside bark obtained by Hohenadl's formula made it possible to compare the bark volumes in percent obtained by Meyer's formula with the observed volumes obtained by the conventional formula. It was observed that Meyer's formula, using only diameter measurments at $\mathrm{DBH}$, underestimated the percent of bark volume for all DBH and ages classes. It was also observed that the ratio between diameter inside bark over diameter outside bark is not constant along the stem. Keywords: Meyer's formula; bark volume; bark factor; Mimosa scabrella.
\end{abstract}

\section{INTRODUÇÃO}

A bracatinga (Mimosa scabrella Bentham) é uma espécie típica dos climas mais frios do sul do Brasil, particularmente no estado do Paraná, onde apresenta áreas expressivas de ocorrência natural (LAURENT et al., 1990). Exclusiva da vegetação secundária da Floresta Ombrófila Mista de formação montana e altomontana, é encontrada principalmente formando comunidades quase puras, conhecidas como bracatingais. Forma agrupamentos densos e é manejada em povoamentos homogêneos com 
finalidade energética e madeireira (BAKES; IRGANG, 2002), especialmente na região metropolitana de Curitiba, onde se constitui em uma importante alternativa econômica no meio rural.

É uma espécie pioneira e pouco exigente quanto às condições do solo, apresentando rápido crescimento. A idade ideal de corte vai depender da necessidade do mercado, da finalidade de aproveitamento da madeira e dos objetivos da empresa. De uma maneira geral, pode-se preconizar a idade de 4 a 7 anos como a de máxima produção biológica (REITZ; KLEIN; REIS, 1988).

EMBRAPA FLORESTA (1988) registrou três variedades: a bracatinga branca, que é a mais abundante e preferida por ser mais fácil de trabalhar; a vermelha, que tem madeira mais dura $\mathrm{e}$ avermelhada, ocorrendo em menor quantidade junto com a variedade branca; e, por fim, a variedade argentina, que tem crescimento mais rápido, folhas mais claras e frutos e sementes maiores e mais rugosos.

Atualmente, o mais expressivo uso da bracatinga é para energia, como lenha e carvão vegetal, embora a literatura também tenha citado com freqüência o uso em construção civil, marcenaria, consórcio com lavouras de subsistência, consórcio com apicultura e como alternativa para recuperação de áreas degradadas.

Apesar das diferentes possibilidades de uso, a bracatinga ainda carece de estudos, especialmente os que trazem informações dendrométricas. Existem espécies cuja madeira é comercializada sem a casca; nesse caso, o volume de casca deve ser descontado. Há também espécies cujo valor comercial reside na própria casca; para estas, o volume da casca tem importância ainda maior, porque está relacionado com a produtividade da espécie.

$\mathrm{O}$ volume da casca é geralmente obtido pela diferença entre o volume com casca e o volume sem casca do tronco. Essa prática algumas vezes é onerosa, dependendo dos propósitos. Uma forma mais prática e interessante de se determinar o volume da casca em porcentagem de uma árvore é através da metodologia desenvolvida por Meyer (1946), que utiliza apenas medições obtidas no nível do DAP, agilizando o trabalho de campo.

No presente trabalho, procurou-se verificar se tal metodologia tem aplicabilidade a bracatingais da região metropolitana de Curitiba, com idades de seis a dezessete anos. Essa metodologia foi descrita por Machado e Garcia (1984), que a testaram em plantações de Pinus taeda, e por Nunes (1981), que a usou em plantações do gênero Eucalyptus. Silva e Paula Neto (1979) afirmaram que a casca pode representar até $25 \%$ do volume com casca da árvore, dependendo da espécie e do local onde ela se encontra. Já para Husch et al. (1982), esse valor chega no máximo a 20\%. Este autor destacou o método do fator de casca $K$ para se determinar o volume de casca em porcentagem e afirmou que tal método produz resultados acurados para a maioria dos propósitos.

O fator de casca é a relação entre diâmetro sem casca sobre diâmetro com casca e foi idealizado por Meyer (1946), que concluiu que $K$ é constante ao longo do fuste e também desenvolveu metodologia para estimar a espessura da casca. Loetsch et al. (1973) afirmaram que a relação entre espessura de casca e DAP depende de influências específicas, como clima, idade e sítio, e que a relação entre diâmetro sem casca e diâmetro com casca pode ser ou não constante ao longo do tronco, dependendo da espécie. Até certa altura a casca é grossa, então mudando abruptamente em algum ponto em direção à extremidade mais fina.

Neste trabalho, testou-se a metodologia de Meyer (1946) para determinar o volume de casca em porcentagem, bem como o fator de casca em bracatingais da região metropolitana de Curitiba, Paraná.

\section{MATERIAIS E MÉTODOS}

\section{Área de estudo}

O trabalho foi realizado em bracatingais formados por regeneração natural pertencentes a diversos proprietários, com idades variando de 6 a 17 anos, localizados em alguns municípios que compõem a região metropolitana de Curitiba, Paraná, mais ao norte da capital, sendo eles: Tunas do Paraná, Rio Branco do Sul, Almirante Tamandaré, Bocaiúva do Sul, Colombo, Campina Grande do Sul, Campo Magro e Itaperuçú. A região encontra-se no primeiro planalto do Paraná, com altitudes variando de 850 a $950 \mathrm{~m}$, clima tipo Cfb segundo classificação de Köpen, onde se registram temperaturas médias anuais em torno de 16 a $17^{\circ} \mathrm{C}$ e a precipitação anual atinge $1500 \mathrm{~mm}$. 


\section{Dados e cálculos}

Foram derrubadas 179 árvores com diâmetros com casca variando de 5 a $32 \mathrm{~cm}$, em bracatingais de 6 a 17 anos. Para cada árvore, foram tomados os diâmetros à altura do peito $\left(\mathrm{DAP}_{1,3}\right)$, com casca e em posições relativas à altura do fuste até a bifurcação, de acordo com o método de cubagem rigorosa de Hohenadl, com divisão do fuste em 10 seções de igual comprimento. Na metade de cada seção, foram medidos os diâmetros com casca, bem como as espessuras da casca. Com esses dados foi possível calcular o volume real com casca $\left(v_{c / c}\right)$ e sem casca $\left(v_{s / c}\right)$ de cada árvore, aplicando-se a fórmula de Hohenadl. Primeiro, as 179 árvores foram agrupadas em classes de diâmetro com $3 \mathrm{~cm}$ de amplitude (Tabela 1). Foi então calculado o volume da casca em porcentagem para cada classe (Tabela 3), pelo método tradicional $\left(v_{r c \%}\right)$ e pela fórmula de Meyer $\left(v_{c \%}\right)$ :

$$
\begin{aligned}
v_{r c \%} & =\frac{\left(\sum_{i=1}^{179} v_{c / c}-\sum_{i=1}^{179} v_{s / c}\right)}{\sum_{i=1}^{179} v_{c / c}} .100 \\
v_{c \%} & =\left(1-K^{2}\right) .100
\end{aligned}
$$

onde $K$ é o resultado da relação de diâmetro sem casca sobre diâmetro com casca ou $K=\frac{\sum_{i=1}^{n} D A_{s c}}{\sum_{i=1}^{n} D A P}$

Essa primeira organização dos dados permitiu visualizar o comportamento da porcentagem de casca com o aumento da classe diamétrica e verificar a acurácia da fórmula de Meyer. Depois, as 179 árvores-amostra foram agrupadas em classes de idade (Tabelas 2 e 4). Isso permitiu observar o comportamento da porcentagem de casca com o aumento da idade. Foram amostradas árvores com as idades de 5, 18 e 19 anos, porém, no momento de agrupá-las em classes de idade, verificou-se que nessas idades não apresentavam um número representativo de indivíduos, sendo deixadas de fora da organização por idade. Com isso, 5 árvores não foram consideradas, e o número total de árvores passou de 179 para 174, distribuídas nas idades de 6 a 17 anos.

De acordo com Meyer, o fator de casca $K$ permanece constante ao longo do fuste, e para verificar se isso ocorre para a bracatinga, aplicou-se a fórmula de Meyer $\left(v_{c \%}\right)$ para medições tomadas a um quarto $(0,25 \mathrm{~h})$, a $0,55 \mathrm{~h}$ e a três quartos $(0,75 \mathrm{~h})$ da altura total, além do DAP. Para traçar linhas estimativas do $v_{c \%}$ em função do DAP, foi preciso encontrar um modelo de equação que melhor se ajustasse à tendência dos dados. Partiu-se do modelo $v_{c \%}=b_{0}+b_{1} d_{i}$ e foi-se acrescentando a variável independente $d$ elevada a diferentes potências até se chegar a um modelo considerado satisfatório. Dessa forma, obteve-se o polinômio $v_{c \%}=b_{0}+b_{1} d_{i}+b_{2} d_{i}^{2}+b_{3} d_{i}^{3}$ onde $d_{i}$ é o diâmetro à altura do peito ( $\left.\mathrm{DAP}_{1,3}\right)$ com casca. A escolha do melhor modelo foi baseada no seu desempenho em relação ao coeficiente de determinação $\left(R^{2}\right)$, ao erro padrão da estimativa expresso como uma porcentagem da média $\left(s_{y x \%}\right)$ e na análise de resíduos. Foram estimados os coeficientes desse modelo para os $v_{c \%}$ tomados no DAP, a $0,25 \mathrm{~h}$, a $0,55 \mathrm{~h}$ e a $0,75 \mathrm{~h}$. Substituindo-se os coeficientes no modelo, resultaram equações as quais foram usadas para traçar linhas estimativas do $v_{c \%}$ em função do DAP, que por sua vez permitiram uma comparação visual dos dados.

\section{Fórmula de Meyer}

Meyer, em 1946, desenvolveu dedutivamente uma fórmula para calcular o volume da casca em porcentagem, baseada apenas na medição dos DAP com casca $(D)$ e DAP sem casca $(d)$. Esse autor 
partiu do princípio de que há uma relação linear muito forte entre $D$ e $d$. A linha de regressão normalmente passa por quase todos os pontos e pela origem, ou muito próximo dela.

Assim:

$$
d=b_{0}+b_{1} \cdot D
$$

Sendo $b_{0}$ aproximadamente igual a zero e substituindo-se $b_{1}$ pela constante $\mathrm{K}$, resulta:

$$
d=K . D \quad \text { (1) } \quad K=\frac{d}{D} \quad K=\frac{\sum_{i=1}^{n} d}{\sum_{i=1}^{n} D}
$$

O volume com casca $\left(v_{c / c}\right)$ e o volume sem casca $\left(v_{s / c}\right)$ do fuste, assumindo-se o mesmo fator de forma, são dados pelas expressões:

$$
\begin{aligned}
& v_{c / c}=\frac{\pi}{4} \cdot d_{c / c}^{2} \cdot h \cdot f \\
& v_{s / c}=\frac{\pi}{4} \cdot d_{s / c}^{2} \cdot h \cdot f
\end{aligned}
$$

Substituindo-se $d$ pelo seu valor em (1), resulta:

$$
v_{s / c}=\frac{\pi}{4} \cdot\left(K \cdot d_{s / c}\right)^{2} \cdot h \cdot f \quad \text { ou } \quad v_{s / c}=\frac{\pi}{4} \cdot\left(K^{2}\right) \cdot d_{s / c}^{2} \cdot h \cdot f
$$

Esta última expressão é igual a $v_{c / c} \cdot K^{2}$, donde resulta:

$$
v_{s / c}=v_{c / c} \cdot K^{2}
$$

Ora:

$$
v_{c}=v_{c / c}-v_{s / c}
$$

Substituindo-se $v_{s / c}$ pelo seu valor em (2), resulta:

$$
\begin{aligned}
& v_{c}=v_{c / c}-v_{c / c} \cdot K^{2} \quad \text { ou } \\
& v_{c}=v_{c / c} \cdot\left(1-K^{2}\right)
\end{aligned}
$$

Sabe-se que o volume real da casca em porcentagem $v_{c \%}$ é dado por:

$$
\begin{aligned}
& v_{c \%}=\frac{\left(v_{c / c}-v_{s / c}\right)}{v_{c / c}} \cdot 100 \\
& v_{c \%}=\frac{\left(v_{c}\right)}{v_{c / c}} \cdot 100
\end{aligned}
$$

Substituindo-se $v_{c}$ pelo seu valor em (3), resulta:

$$
v_{c \%}=\frac{v_{c / c} \cdot\left(1-K^{2}\right)}{v_{c / c}} \cdot 100
$$


Simplificando, resulta finalmente a equação (4):

$$
v_{c \%}=\left(1-K^{2}\right) \cdot 100
$$

Essa é a fórmula final de Meyer (1946) para calcular o volume da casca em porcentagem, sem necessidade de calcular volumes, com e sem casca, e que será testada para a bracatinga neste trabalho.

\section{RESULTADOS E DISCUSSÃO}

\section{Volume de casca em porcentagem}

Com os dados individuais dos diâmetros à altura do peito com casca $\left(\mathrm{DAP}_{\mathrm{c} / \mathrm{c}}\right)$ e sem casca $\left(\mathrm{DAP}_{\mathrm{s} / \mathrm{c}}\right)$ de cada uma das 179 árvores amostradas e também seus respectivos volumes com casca $\left(v_{c / c}\right)$ e sem casca $\left(v_{s / c}\right)$, decidiu-se primeiro agrupar esses dados por classe de DAP, com intervalo de 3 centímetros. Esses mesmos dados foram também agrupados por classes de idade com intervalo de 2 anos. As tabelas 1 e 2 mostram os resultados das somatórias dos DAP, volumes e também o número de árvores em cada classe.

Os dados agrupados dessas tabelas serviram como base para o cálculo do volume de casca em porcentagem pela fórmula de Meyer $\left(v_{c \%}\right)$ e pelo método tradicional $\left(v_{r c \%}\right)$. Os resultados são mostrados nas tabelas 3 e 4.

Tabela 1. Volumes reais com e sem casca por classe de DAP.

Table 1. Observed volumes outside and inside bark by diameter classes.

\begin{tabular}{ccccccc}
\hline $\begin{array}{c}\text { Classe de } \\
\mathbf{D A P} \\
\mathbf{( c m )}\end{array}$ & $\begin{array}{c}\text { Centro de } \\
\text { classe } \\
\mathbf{( c m )}\end{array}$ & $\begin{array}{c}\text { Número } \\
\mathbf{d e} \\
\text { árvores }\end{array}$ & $\begin{array}{c}\sum_{i=1}^{179} D A P_{c / c} \\
\mathbf{( c m})\end{array}$ & $\begin{array}{c}\sum_{i=1}^{179} D A P_{s / c} \\
\mathbf{( c m )}\end{array}$ & $\begin{array}{c}\sum_{i=1}^{179} v_{c / c} \\
\left(\mathbf{m}^{3}\right)\end{array}$ & $\begin{array}{c}\sum_{i=1}^{179} v_{s / c} \\
\left(\mathbf{m}^{3}\right)\end{array}$ \\
\hline $5-7,9$ & 6,5 & 13 & 87,50 & 80,60 & 0,2849 & 0,2389 \\
$8-10,9$ & 9,5 & 20 & 185,90 & 172,90 & 0,9620 & 0,8251 \\
$11-13,9$ & 12,5 & 34 & 422,30 & 394,40 & 3,0143 & 2,6246 \\
$14-16,9$ & 15,5 & 35 & 531,06 & 496,66 & 4,6549 & 4,0524 \\
$17-19,9$ & 18,5 & 27 & 494,55 & 463,49 & 4,8964 & 4,2604 \\
$20-22,9$ & 21,5 & 21 & 454,95 & 426,75 & 5,2394 & 4,5334 \\
$23-25,9$ & 24,5 & 16 & 394,45 & 368,45 & 5,5729 & 4,8267 \\
$26-28,9$ & 27,5 & 9 & 243,55 & 226,75 & 3,5035 & 3,0059 \\
$29-32$ & 30,5 & 4 & 121,15 & 114,15 & 1,8338 & 1,5928 \\
\hline Total & - & 179 & - & - & - & - \\
\hline
\end{tabular}

Tabela 2. Volumes reais com e sem casca por classe de idade.

Table 2. Observed volumes outside and inside bark by age classes.

\begin{tabular}{ccccccc}
\hline $\begin{array}{c}\text { Idade } \\
\text { (anos) }\end{array}$ & $\begin{array}{c}\text { Centro de } \\
\text { classe } \\
\mathbf{( c m )}\end{array}$ & $\begin{array}{c}\text { Número de } \\
\text { árvores }\end{array}$ & $\begin{array}{c}\sum_{i=1}^{174} D A P_{c / c} \\
\mathbf{( c m})\end{array}$ & $\begin{array}{c}\sum_{i=1}^{174} D A P_{s / c} \\
\mathbf{( c m )}\end{array}$ & $\begin{array}{c}\sum_{i=1}^{174} v_{c / c} \\
\left(\mathbf{m}^{3}\right)\end{array}$ & $\begin{array}{c}\sum_{i=1}^{174} v_{s / c} \\
\left(\mathbf{m}^{3}\right)\end{array}$ \\
\hline $6-7$ & 6,5 & 13 & 139,20 & 130,50 & 0,7906 & 0,6868 \\
$8-9$ & 8,5 & 35 & 384,30 & 358,20 & 2,3509 & 2,0247 \\
$10-11$ & 10,5 & 56 & 865,11 & 811,61 & 7,9379 & 6,9488 \\
$12-13$ & 12,5 & 38 & 738,60 & 691,40 & 8,2573 & 7,1391 \\
$14-15$ & 14,5 & 17 & 330,75 & 307,39 & 3,6784 & 3,1620 \\
$16-17$ & 16,5 & 15 & 367,45 & 342,65 & 5,2062 & 4,4810 \\
\hline Total & - & 174 & - & - & - & - \\
\hline
\end{tabular}


Tabela 3. Volume de casca em porcentagem calculado pela fórmula de Meyer $\left(v_{c \%}\right)$ e pelo método tradicional $\left(v_{r c \%}\right)$ para as classes de diâmetro.

Table 3. Bark volume in percent calculated by the Meyer's formula $\left(v_{c \%}\right)$ and by the traditional method $\left(v_{r c \%}\right)$ by diameter classes.

\begin{tabular}{ccccc}
\hline $\begin{array}{c}\text { Centro de } \\
\text { classe } \\
\text { (cm) }\end{array}$ & $K_{1,3}=\frac{\sum_{i=1}^{179} D A P_{/ c}}{\sum^{179} D A P_{/ c}}$ & & & \\
& $\sum_{i=1}^{2}$ & & & \\
\hline 6,5 & 0,9211 & 0,8485 & 15,1496 & 16,1539 \\
9,5 & 0,9301 & 0,8650 & 13,4970 & 14,2296 \\
12,5 & 0,9339 & 0,8722 & 12,7769 & 12,9275 \\
15,5 & 0,9352 & 0,8746 & 12,5355 & 12,9414 \\
18,5 & 0,9372 & 0,8783 & 12,1665 & 12,9878 \\
21,5 & 0,9380 & 0,8799 & 12,0128 & 13,4734 \\
24,5 & 0,9341 & 0,8725 & 12,7484 & 13,3895 \\
27,5 & 0,9310 & 0,8668 & 13,3201 & 14,2025 \\
30,5 & 0,9422 & 0,8878 & 11,2221 & 13,1387 \\
\hline
\end{tabular}

Tabela 4. Volume de casca em porcentagem calculado pela fórmula de Meyer $\left(v_{c \%}\right)$ e pelo método tradicional $\left(v_{r c \%}\right)$ para as classes de idade.

Table 4. Bark volume in percent calculated by th Meyer's formula $\left(v_{c \%}\right)$ and by the traditional method $\left(v_{r c \%}\right)$ by age classes.

\begin{tabular}{|c|c|c|c|c|}
\hline $\begin{array}{l}\text { Idade } \\
\text { (anos) }\end{array}$ & $=\frac{\sum_{i=1}^{174} D A}{\sum_{i=1}^{17} D A}$ & $K^{2}$ & $v_{c \%}=\left(1-K^{2}\right) \cdot 100$ & $v_{r c \%}$ \\
\hline 6,5 & 0,9375 & 0,8789 & 12,1094 & 13,1324 \\
\hline 8,5 & 0,9321 & 0,8688 & 13,1219 & 13,8791 \\
\hline 10,5 & 0,9382 & 0,8801 & 11,9859 & 12,4602 \\
\hline 12,5 & 0,9361 & 0,8763 & 12,3726 & 13,5409 \\
\hline 14,5 & 0,9294 & 0,8637 & 13,6266 & 14,0387 \\
\hline 16,5 & 0,9325 & 0,8696 & 13,0429 & 13,9290 \\
\hline
\end{tabular}

Observando a tabela 3, verifica-se que o fator de casca $\mathrm{K}$ variou de 0,92 a 0,94 , resultando numa variação de volume da casca em porcentagem $\left(v_{c \%}\right)$ de $15,1 \%$ a $11,2 \%$ para as classes diamétricas, variação esta bem menor do que a encontrada por Machado e Garcia (1984), que foi de 23\% a $12 \%$ para Pinus taeda. Essa pequena variação da porcentagem de casca da classe menor para a maior (3,9\%) levou a suspeitar que poderia tratar-se de uma flutuação biológica natural e que a porcentagem de casca poderia ser considerada aproximadamente constante ao longo das classes de DAP para a bracatinga.

Através da tabela 4, percebe-se uma variação no valor de K ainda menor $(0,92$ a 0,93$)$ e conseqüentemente uma variação de $v_{c \%}$ de $11,9 \%$ a $13,6 \%$, ou seja, menos que $2 \%$ no volume de casca em porcentagem ao longo das idades. Isso só aumentou a expectativa para um resultado positivo da aplicação da metodologia desenvolvida por Meyer.

Para verificar se realmente a porcentagem de casca foi constante ao longo das classes de DAP e idade e também a acuracidade da fórmula de Meyer $\left(v_{c \%}\right)$ em relação ao volume real de casca em porcentagem $\left(v_{r c \%}\right)$, decidiu-se aplicar uma análise de variância em blocos casualizados para as classes 
de DAP e também para as classes de idade, na qual as classes de DAP e idades foram os blocos e os métodos usados para obtenção da porcentagem de casca $\left(v_{c \%}\right.$ e $\left.v_{r c \%}\right)$ foram considerados os tratamentos.

Preliminarmente, aplicou-se o teste de Barttlet para verificar se as variâncias são homogêneas, para então proceder-se a análise de variância propriamente dita. O qui-quadrado do teste de Barttlet para um nível de 0,01 de probabilidade, quando os dados foram agrupados em classe de diâmetro, foi de 0,029 contra um qui-quadrado tabelado de 6,63, portanto não significativo. O mesmo ocorreu quando os dados foram agrupados em classes de idade, para o mesmo nível de probabilidade, sendo o qui-quadrado igual a 0,010 contra um qui-quadrado tabelado de 6,63. Concluiu-se então que as variâncias dentro das classes de $v_{c \%}$ e $v_{r c \%}$ não foram significativas a uma probabilidade de $99 \%$, o que quer dizer que as variâncias dos dois grupos ( $v_{c \%}$ e $v_{r c \%}$ ) são homogêneas, tanto para os dados agrupados em classe de diâmetro quanto para os dados agrupados em classes de idade. Essa é a condição básica para que possa ser aplicada a análise de variância.

Tabela 5. Resultado da análise de variância em blocos casualizados.

Table 5. Analysis of variance results for random blocks design.

\begin{tabular}{lcccc|cccc}
\hline \multirow{2}{*}{$\begin{array}{l}\text { Fonte de } \\
\text { variação }\end{array}$} & \multicolumn{4}{c}{ Classe de DAP } & \multicolumn{4}{c}{ Classe de idade } \\
\cline { 2 - 8 } & G.L. & FO & FT 0,05 & FT 0,01 & G.L. & FO & FT 0,05 & FT 0,01 \\
\hline Blocos & 8 & $15,419^{* *}$ & 3,34 & 6,03 & 5 & $16,58^{* *}$ & 6,61 & 16,26 \\
Tratamento & 1 & $25,270^{* *}$ & 5,32 & 11,26 & 1 & $41,259^{* *}$ & 5,05 & 10,97 \\
Erro & 8 & & & & & 5 & & \\
\hline
\end{tabular}

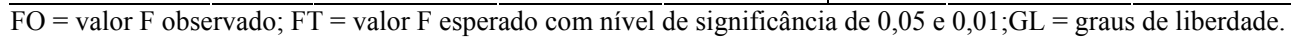

Na análise de variância, testou-se a hipótese de que não há diferença entre os resultados obtidos através da fórmula de Meyer $\left(v_{c \%}\right)$ e os obtidos através da cubagem das árvores $\left(v_{r c \%}\right)$, tanto para as classes de diâmetro quanto para as classes de idade. Os resultados mostraram que o valor de $\mathrm{F}$ observado (FO) para os tratamentos foi significante ao nível de 0,01 de significância. Isso quer dizer que a fórmula de Meyer não propiciou resultados estatisticamente iguais aos reais para o volume de casca em porcentagem.

Machado e Garcia (1984) observaram que os resultados obtidos através da fórmula de Meyer superestimaram bastante o volume de casca em porcentagem em todas as classes de diâmetro para Pinus taeda. Neste trabalho, observou-se que tal fórmula subestimou o referido volume para todas as classes de diâmetro e que essa diferença para o real foi significativa.

Nunes (1981) verificou tendência de diminuição da porcentagem de casca com o aumento da idade para três espécies de Eucalyptus. No caso da bracatinga, houve uma aparente constância na porcentagem de casca com o aumento da idade, porém a análise de variância mostrou que as diferenças na porcentagem de casca entre as idades não permitem considerá-la constante.

\section{Modelo para traçar as linhas estimativas do $v_{c \%}$ em função do DAP}

Os volumes da casca em porcentagem foram estimados através de equações de regressão linear desenvolvidas usando-se os $v_{c \%}$ e $v_{r c \%}$ calculados como variável dependente e os centros de classes de diâmetro como variável independente. Partiu-se do modelo $v_{c \%}=b_{0}+b_{1} d_{i}$ e foi-se acrescentando a variável independente diâmetro $(d)$ até resultar no modelo $v_{c \%}=b_{0}+b_{1} d_{i}+b_{2} d_{i}^{2}+b_{3} d_{i}^{3}$, que apresentou melhor desempenho com base no coeficiente de determinação $\left(R^{2}\right)$, no erro padrão da estimativa em porcentagem $\left(s_{y x \%}\right)$ e na análise dos resíduos $(E \%)$. Como a fórmula de Meyer $\left(v_{c \%}\right)$ forneceu resultados próximos do real $\left(v_{r c \%}\right)$, o melhor modelo para $v_{r c \%}$ foi também o melhor para $v_{c \%}$. Os modelos escolhidos, com seus respectivos ajustes e precisão para as duas situações, são os seguintes:

$$
\text { a) } \begin{gathered}
\hat{v}_{r c \%}=25,315590-2,002511 d_{i}+0,102373 d_{i}^{2}-0,001628 d_{i}^{3} \\
R^{2}=0,9537 \\
S_{y x \%}=2,0661
\end{gathered}
$$




$$
\text { b) } \begin{gathered}
\hat{v}_{c \%}=23,251455-1,762006 d_{i}+0,0091562 d_{i}^{2}-0,001517 d_{i}^{3} \\
R^{2}=0,85871 \\
s_{y x \%}=4,1146
\end{gathered}
$$

A tabela 6 mostra o volume de casca em porcentagem calculado pelo método convencional $\left(v_{r c \%}\right)$ e pela fórmula de Meyer $\left(v_{c \%}\right)$ e seus respectivos valores estimados pelos modelos ajustados juntamente com o resíduo $(E \%)$ gerado para cada um deles.

Tabela 6. Volume da casca em porcentagem calculados $\left(v_{r c \%} ; v_{c \%}\right)$ e estimados pelos modelos ajustados $\left(\hat{v}_{r c \%} ; \hat{v}_{c \%}\right)$ e seus respectivos resíduos.

\begin{tabular}{|c|c|c|c|c|c|c|c|}
\hline $\begin{array}{c}\text { Classe de } \\
\text { DAP } \\
\text { (cm) }\end{array}$ & $\begin{array}{l}\text { Centro de } \\
\text { classe } \\
\text { (cm) }\end{array}$ & $v_{r c \%}$ & $\hat{v}_{r c \%}$ & $\begin{array}{c}E \% \\
\text { relativo a } \\
v_{r c \%}\end{array}$ & $v_{c \%}=\left(1-K^{2}\right) \cdot 100$ & $\hat{v}_{c \%}$ & $\begin{array}{c}E \% \\
\text { relativo } \\
\text { a } v_{c \%}\end{array}$ \\
\hline $5-8$ & 6,5 & 16,1539 & 16,1818 & $-0,1731$ & 15,1496 & 15,2541 & $-0,6901$ \\
\hline $8-11$ & 9,5 & 14,2296 & 14,1376 & 0,6465 & 13,4970 & 13,4774 & 0,1452 \\
\hline $11-14$ & 12,5 & 12,9275 & 13,1013 & $-1,3448$ & 12,7769 & 12,5710 & 1,6115 \\
\hline $14-17$ & 15,5 & 13,0064 & 12,8093 & 1,5151 & 12,5355 & 12,2891 & 1,9657 \\
\hline $17-20$ & 18,5 & 12,9278 & 12,9978 & $-0,5415$ & 12,1665 & 12,3860 & $-1,8045$ \\
\hline $20-23$ & 21,5 & 13,4734 & 13,4031 & 0,5217 & 12,0128 & 12,6160 & $-5,0216$ \\
\hline $23-26$ & 24,5 & 13,3895 & 13,7615 & $-2,7778$ & 12,7484 & 12,7332 & 0,1192 \\
\hline $26-29$ & 27,5 & 14,2025 & 13,8091 & 2,7699 & 13,3201 & 12,4920 & 6,2168 \\
\hline $29-32$ & 30,5 & 13,1387 & 13,2823 & $-1,0930$ & 11,2221 & 11,6466 & $-3,7830$ \\
\hline
\end{tabular}

Table 6. Calculated bark volumes in percent $\left(v_{r c \%} ; v_{c \%}\right)$ and estimated ones by the fited models $\left(\hat{v}_{r c \%}, \hat{v}_{c} \%\right)$ and their respectives residuals.

\section{Volume da casca em diferentes alturas}

Segundo a mesma metodologia utilizada para o cálculo do volume da casca em porcentagem com dados do DAP, foram feitos também os cálculos para os volumes da casca em porcentagem com base nos diâmetros tomados a alturas relativas de $25 \%, 55 \%$ e $75 \%$ da altura total ou $0,25 \mathrm{~h}, 0,55 \mathrm{~h}$ e $0,75 \mathrm{~h}$. Com isso, pode-se verificar se o volume da casca em porcentagem permanece constante ao longo do fuste, como encontrou Meyer, citado por Machado e Garcia (1984). Os resultados são apresentados na tabela 7.

Os volumes de casca em porcentagem nas diferentes alturas, conforme consta na tabela 7, foram submetidos também a uma análise de variância em blocos casualizados para verificar se há uma constância entre os valores. Os referidos volumes foram considerados como tratamentos e as classes de diâmetro como blocos. Os resultados aparecem na tabela 8 . Antes da análise de variância, verificou-se se os volumes de casca em porcentagem eram homogêneos, através do teste de Barttlet. O qui-quadrado observado pelo teste de Barttlet foi de 0,740 contra um qui-quadrado esperado de 9,21 para um nível de significância de $1 \%$, o que evidenciou que as variâncias dos volumes de casca em porcentagem são homogêneas. $\mathrm{O}$ resultado da análise de variância para $v_{c \%}$ em diferentes alturas forneceu os seguintes resultados:

Conforme resultados da análise de variância apresentados na tabela 8 , a hipótese de que a porcentagem de casca é constante ao longo do fuste foi rejeitada. O valor de $\mathrm{F}$ observado (FO) do tratamento foi significativo a um de nível de significância de $1 \%$, o que equivale a dizer que o fator de casca $\mathrm{K}$ não foi constante ao longo do tronco.

$\mathrm{O}$ modelo ajustado anteriormente para os dados tomados à altura do peito (DAP) foi também ajustado para cada uma das alturas relativas mencionadas anteriormente. Os resultados aparecem na tabela 9, onde foram colocados também os resultados das estimativas obtidas no nível do DAP. A partir 
dessa tabela, traçaram-se as linhas estimativas em relação ao DAP, mostradas na figura 1. Na tabela 10, aparece o resumo dos coeficientes das equações resultantes, bem como o $R^{2}$ e $S_{y x \%}$.

Tabela 7. Fator de casca $\mathrm{K}$ em diferentes alturas e os conseqüentes volumes percentuais de casca $\left(v_{c \%}\right)$ calculados pela fórmula de Meyer.

Table 7. Bark factor $\mathrm{K}$ at different heights and the correspondent bark volumes in percent $\left(v_{c \%}\right)$ calculated by Meyer's formula.

\begin{tabular}{|c|c|c|c|c|c|c|c|}
\hline $\begin{array}{c}\text { Classe de } \\
\text { DAP } \\
(\mathrm{cm})\end{array}$ & $\begin{array}{c}\text { Centro de } \\
\text { classe } \\
(\mathrm{cm})\end{array}$ & $\begin{array}{c}K \text { a } \\
\mathbf{0 , 2 5 h}\end{array}$ & $\begin{array}{c}v_{c \%} \\
\text { a } \mathbf{0 , 2 5 h}\end{array}$ & $\begin{array}{c}K \text { a } \\
\mathbf{0 , 5 5 h}\end{array}$ & $\begin{array}{c}v_{c \%} \\
\text { a } \mathbf{0 , 5 5 h}\end{array}$ & $\begin{array}{c}K \text { a } \\
\mathbf{0 , 7 5 h}\end{array}$ & $\begin{array}{c}v_{c \%} \\
\text { a } 0,75 \mathbf{h}\end{array}$ \\
\hline $5-8$ & 6,5 & 0,9222 & 14,9629 & 0,9149 & 16,2915 & 0,9108 & 17,0392 \\
\hline $8-11$ & 9,5 & 0,9311 & 13,3080 & 0,9281 & 13,8630 & 0,9226 & 14,8766 \\
\hline $11-14$ & 12,5 & 0,9341 & 12,7536 & 0,9314 & 13,2534 & 0,9256 & 14,3266 \\
\hline $14-17$ & 15,5 & 0,9327 & 13,0062 & 0,9305 & 13,4179 & 0,9275 & 13,9647 \\
\hline $17-20$ & 18,5 & 0,9339 & 12,7873 & 0,9326 & 13,0332 & 0,9301 & 13,4826 \\
\hline $20-23$ & 21,5 & 0,9365 & 12,2946 & 0,9321 & 13,1213 & 0,9275 & 13,9684 \\
\hline $23-26$ & 24,5 & 0,9332 & 12,9192 & 0,9282 & 13,8350 & 0,9252 & 14,3925 \\
\hline $26-29$ & 27,5 & 0,9288 & 13,7280 & 0,9238 & 14,6676 & 0,9191 & 15,5253 \\
\hline $29-32$ & 30,5 & 0,9439 & 10,9112 & 0,9285 & 13,7899 & 0,9163 & 16,0423 \\
\hline
\end{tabular}

Tabela 8. Análise de variância para volume de casca em porcentagem em diferentes alturas.

Table 8. Analysis of variance table for bark volume in percent at different heights.

\begin{tabular}{lcccc}
\hline \multirow{2}{*}{ Fonte de variação } & \multicolumn{4}{c}{ Classe de DAP } \\
\cline { 2 - 5 } & G.L. & FO & FT 0,05 & FT 0,01 \\
\hline Blocos & 8 & $6,247^{* *}$ & 3,63 & 6,23 \\
Tratamento & 2 & $18,565^{* *}$ & 2,59 & 3,89 \\
Erro & 16 & & & \\
\hline
\end{tabular}

Tabela 9. Estimativas do volume de casca em porcentagem para o DAP e em diferentes alturas.

Table 9. Bark volume estimates in percent at DBH level and at different relative heights along the tree stem.

\begin{tabular}{cccccc}
\hline $\begin{array}{c}\text { Classe de } \\
\text { diâmetro } \\
(\mathbf{c m})\end{array}$ & $\begin{array}{c}\hat{v}_{r c \%} \\
\mathrm{a} 1,30 \mathrm{~m}\end{array}$ & $\begin{array}{c}\hat{v}_{c \%} \\
\mathrm{a} 1,30 \mathrm{~m}\end{array}$ & $\begin{array}{c}\hat{v}_{c \%} \\
\mathrm{a} 0,25 \mathrm{~h}\end{array}$ & $\begin{array}{c}\hat{v}_{c \%} \\
\mathrm{a} 0,55 \mathrm{~h}\end{array}$ & $\begin{array}{c}\hat{v}_{c \%} \\
\mathrm{a} 0,75 \mathrm{~h}\end{array}$ \\
\hline $5-8$ & 16,1817 & 15,2570 & 14,9960 & 16,1577 & 16,9126 \\
$8-11$ & 14,1372 & 13,4827 & 13,3948 & 14,2199 & 15,2209 \\
$11-14$ & 13,1006 & 12,5712 & 12,6818 & 13,2179 & 14,1797 \\
$14-17$ & 12,8078 & 12,2800 & 12,5773 & 12,9214 & 13,6911 \\
$17-20$ & 12,9953 & 12,3668 & 12,8012 & 13,1006 & 13,6572 \\
$20-23$ & 13,3992 & 12,5893 & 13,0738 & 13,5254 & 13,9801 \\
$23-26$ & 13,7556 & 12,7050 & 13,1153 & 13,9657 & 14,5618 \\
$26-29$ & 13,8008 & 12,4717 & 12,6457 & 14,1915 & 15,3045 \\
$29-32$ & 13,2710 & 11,6469 & 11,3851 & 13,9727 & 16,1101 \\
\hline
\end{tabular}

Para facilitar o entendimento da figura 1, preparou-se a tabela 11, para visualização dos valores médios resultantes das medições em diferentes alturas. 
Tabela 10. Coeficientes e estatísticas das equações de regressão para a estimativa do volume da casca em porcentagem em diferentes alturas ao longo do fuste.

Table 10. Coefficients and statistics of the regression equations for bark volume estimate in percent at different heights along the tree stem.

\begin{tabular}{cccccccc}
\hline Alturas & $b_{0}$ & $b_{1}$ & $b_{2}$ & $b_{3}$ & $R^{2}$ & $s_{y x}$ & $s_{y x \%}$ \\
\hline $\operatorname{DAP}_{1,3}\left(v_{r c \%}\right)$ & 25,31559 & $-2,00251$ & 0,10237 & $-0,00163$ & 0,95370 & 0,28341 & 2,06610 \\
$\operatorname{DAP}_{1,3}\left(v_{c \%}\right)$ & 23,25146 & $-1,76201$ & 0,09156 & $-0,00152$ & 0,85871 & 0,52771 & 4,11460 \\
$0,25 \mathrm{~h}$ & 22,83693 & $-1,77447$ & 0,09856 & $-0,00173$ & 0,76270 & 0,66920 & 5,16289 \\
$0,55 \mathrm{~h}$ & 24,65299 & $-1,84840$ & 0,09243 & $-0,00142$ & 0,89940 & 0,40941 & 2,94137 \\
$0,75 \mathrm{~h}$ & 23,26971 & $-1,29982$ & 0,05336 & $-0,00060$ & 0,96753 & 0,26217 & 1,76587 \\
\hline
\end{tabular}

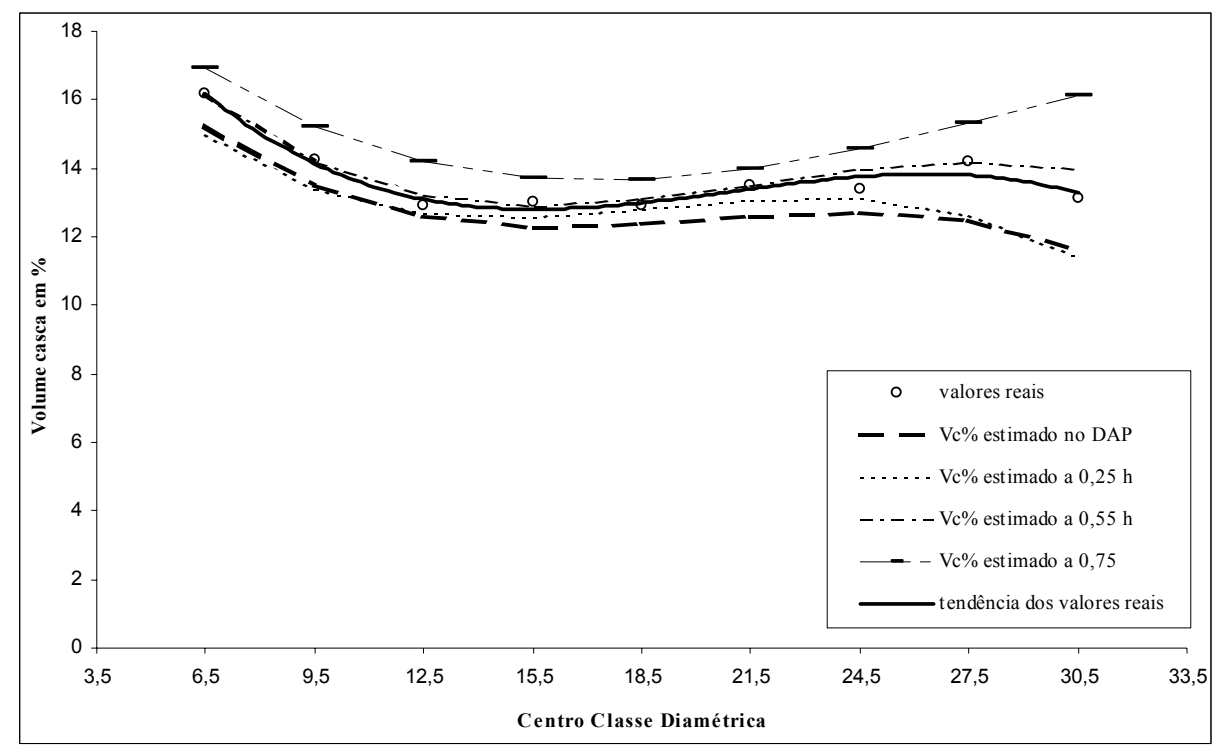

Figura 1. Linhas de tendência dos volumes da casca em porcentagem estimadas em diferentes alturas do fuste, em relação às classes de diâmetro.

Figure 1. Bark volume estimated lines in percent at different heights along the stem in relation to diameter classes.

Como se observa na figura 1 , a linha estimativa com os dados tomados no DAP subestimou o $v_{c \%}$ em todas as classes de diâmetro, o mesmo acontecendo, embora em menor magnitude, com a curva do $v_{c \%}$ estimada com base nos diâmetros tomados a $0,25 \mathrm{~h}$. A curva calculada com base nos diâmetros tomados a $0,75 \mathrm{~h}$ sempre superestimou os $v_{c \%}$, enquanto que a curva do $v_{c \%}$ baseada em diâmetros tomados a $0,55 \mathrm{~h}$ resultou nas melhores estimativas para todas as classes de DAP.

Tabela 11. Valores médios resultantes de medições em diferentes alturas do fuste.

Table 11. Average $K$ and $v_{c \%}$ resulting from measurement at different heights.

\begin{tabular}{lccc}
\hline $\begin{array}{l}\text { Posição de } \\
\text { medição }\end{array}$ & $K$ médio & $K^{2}$ médio & $\begin{array}{c}v_{c \%}=\left(1-K^{2}\right) \cdot 100 \\
\text { médio }\end{array}$ \\
\hline $1,3 \mathrm{~m}$ & 0,9336 & 0,8717 & 12,83 \\
$0,25 \mathrm{~h}$ & 0,9329 & 0,8703 & 12,97 \\
$0,55 \mathrm{~h}$ & 0,9278 & 0,8608 & 13,92 \\
$0,75 \mathrm{~h}$ & 0,9228 & 0,8515 & 14,85 \\
\hline
\end{tabular}

Volume médio real da casca em porcentagem $=13,72$ 
Como pode-se observar pelos dados da tabela 11, o volume médio da casca em porcentagem calculado através de medições de diâmetros com e sem casca a 55\% da altura total da árvore foi o que mais se aproximou do volume médio real da casca em porcentagem calculado pelo método convencional. Machado e Garcia (1984) encontraram que a melhor altura de medição para esse fim em Pinus taeda no Paraná foi a $0,25 \mathrm{~h}$.

\section{CONCLUSÃO}

Com base nos resultados da presente pesquisa, pode-se concluir que:

- O fator de casca $K$ não é constante ao longo do fuste da árvore, o que implica dizer que o método de Meyer para determinação do volume de casca com base em medição do diâmetro com casca e sem casca à altura de $1,30 \mathrm{~m}$ subestimou esse volume e as análises estatísticas mostraram que a diferença foi significativa para todas as classe de DAP e de idade.

- Dentre as alturas relativas testadas, a de $0,55 \mathrm{~h}$ foi a que se apresentou mais adequada para a aplicação do método de Meyer, pois foi a que mostrou resultados mais próximos dos valores observados.

\section{REFERÊNCIAS}

BAKES, P.; IRGANG, B. Árvores do sul. 1ª ed, Rio Grande do Sul, 2002, 326p.

EMBRAPA FLORESTAS. Manual técnico da bracatinga (Mimosa scabrella). Colombo, PR., 1988, $70 \mathrm{p}$.

HUSCH, B.; MILLER, C. I.; BEERS, T. W. Forest mensuration. $3^{\text {a }}$ ed. John Wiley \& Sons. New York, 1982. 402p

LAURENT, J. E.; CAMPOS, J. B.; BITTENCOURT, S. M. de. Análise técnico-econômica do sistema agroflorestal da bracatinga na Região Metropolitana de Curitiba. Série Estudos Florestais n. ${ }^{\circ} 4$. PDFI/FAO, Curitiba, Pr, 1990, 72p.

LOETSCH, F.; ZÖHRER, F.; HALLER, K. E. Forest inventory. München: BLV. Verlagsgesellchaft, 1973, v2, 469p.

MACHADO, S. A.; GARCIA, E. R. Determinação do volume da casca em plantações de Pinus taeda. Revista Floresta, Vol XV, n. ${ }^{\circ} 1$ e 2, Curitiba, Pr, 1984 p.17-25.

MEYER, H. A. Bark Volume determination in trees. Journal of Forestry 44 (12). 1946, p.1067-1070.

NUNES, J. R. S. Análise do volume e da porcentagem de casca em povoamentos de eucaliptos de origem híbrida, segundo a idade, local, espécie e método de regeneração. 103f. Dissertação (mestrado em Ciência Florestal), Universidade Federal de Viçosa, MG, 1981.

REITZ, R.; KLEIN, R. M.; REIS, A. Projeto madeira do Rio Grande do Sul. Herbário Barbosa Rodrigues, Itajaí, 1988, 525p.

SILVA, J. A. A da; PAUlA NETO, F. de. Princípios básicos de dendrometria. Recife: Universidade Federal Rural de Pernambuco, 1979. 185p. 